\title{
A Measurement of Cosmic Ray Deuterium from 0.5-2.9 GeV/nucleon
}

\author{
G.A. de Nolfo ${ }^{1}$, L.M. Barbier ${ }^{2}$, E.R. Christian ${ }^{2}$, A.J. Davis ${ }^{1}$, R.L. Golden ${ }^{5}$, M. Hof ${ }^{4}$, \\ K.E. Krombel ${ }^{2}$, A.W. Labrador ${ }^{6}$, W. Menn ${ }^{4}$, R.A. Mewaldt ${ }^{1}$, J.W. Mitchell ${ }^{2}$, \\ J.F. Ormes ${ }^{2}$, I.L. Rasmussen ${ }^{7}$, O. Reimer ${ }^{3}$, S.M. Schindler ${ }^{1}$, M. Simon ${ }^{4}$, \\ S.J. Stochaj ${ }^{5}$, R.E. Streitmatter ${ }^{2}$, W.R. Webber ${ }^{5}$ \\ ${ }^{1}$ California Institute of Technology, Pasadena, California 91125 \\ ${ }^{2}$ NASA/Goddard Space Flight Center, Greenbelt, MD 20771 \\ ${ }^{3}$ Max-Planck-Institut fuer extraterrestrische Physik, Garching, Germany \\ ${ }^{4}$ Universitat Siegen, Siegen, Germany \\ ${ }^{5}$ New Mexico State University, Las Cruces, NM 88003 \\ ${ }^{6}$ University of Chicago, Chicago, IL 60637 \\ ${ }^{7}$ Danish Space Research Institute, Copenhagen, Denmark
}

\begin{abstract}
The rare isotopes ${ }^{2} \mathrm{H}$ and ${ }^{3} \mathrm{He}$ in cosmic rays are believed to originate mainly from the interaction of high energy protons and helium with the galactic interstellar medium. The unique propagation history of these rare isotopes provides important constraints on galactic cosmic ray source spectra and on models for their propagation within the Galaxy. Hydrogen and helium isotopes were measured with the balloon-borne experiment, IMAX, which flew from Lynn Lake, Manitoba in 1992. The energy spectrum of deuterium between 0.5 and $3.2 \mathrm{GeV} /$ nucleon measured by the IMAX experiment as well as previously published results of ${ }^{3} \mathrm{He}$ from the same instrument will be compared with predictions of cosmic ray galactic propagation models. The observed composition of the light isotopes is found to be generally consistent with the predictions of the standard Leaky Box Model derived to fit observations of heavier nuclei.
\end{abstract}

\section{INTRODUCTION}

Extensive observations of cosmic ray abundances over a wide range in energy help to form a comprehensive picture of cosmic ray origin and propagation. In the simplest picture such as the standard Leaky Box Model, cosmic rays propagate within the Galaxy influenced by the competing processes of nuclear interactions and escape from the Galaxy. The light isotopes such as $\mathrm{Li}$, $\mathrm{Be}, \mathrm{B}$ are significantly enhanced over solar system abundances indicating that these elements are produced as secondary or spallation products of primary $\mathrm{C}, \mathrm{N}$, and $\mathrm{O}$ elements. Thus, the determination of the secondary/primary ratio provides a measure of the amount of material traversed by primary cosmic rays during propagation. At 1 $\mathrm{GeV} /$ nucleon, the mean free pathlength for escape from the Galaxy is found to be $\lambda \sim 10 \mathrm{~g} / \mathrm{cm}^{2}$.

The isotopes ${ }^{2} \mathrm{H}$ and ${ }^{3} \mathrm{He}$ are of particular interest as these isotopes are considered to be interaction products of the more abundant hydrogen and helium nuclei. In addition, the abundance of ${ }^{2} \mathrm{H}$ and ${ }^{3} \mathrm{He}$ can, in principle, provide a more sensitive determination of the escape pathlength than heavier cosmic ray nuclei, since $\mathrm{H}$ and $\mathrm{He}$ are affected by fewer nuclear destruction processes during propagation. These isotopes may also provide a test of whether cosmic rays undergo continuous acceleration or "reacceleration" during their passage through the Galaxy (8). Hydrogen and helium isotopes have been measured by the Isotope Matter-Antimatter Experiment (IMAX) instrument over a wide range in energies extending to 2.9 $\mathrm{GeV} /$ nucleon (6). In this paper, we present IMAX measurements of ${ }^{2} \mathrm{H}$ as well as the previously published results of ${ }^{3} \mathrm{He}(6)$ and compare these observations with predictions from current propagation models.

\section{INSTRUMENT AND FLIGHT}

IMAX was designed to measure antiprotons and the light isotopes over a wide energy range. IMAX employed a combination of detectors including a superconducting magnetic spectrometer (2), a time-of-flight (TOF) system, scintillation counters (S1,S2), and large-area aerogel Cherenkov detectors. Particle identification is accomplished by measuring the particle velocity $\beta$, charge $Z$, and rigidity $\mathrm{R}$ (momentum/charge). For further details on the performance of the IMAX instrument see (1). 

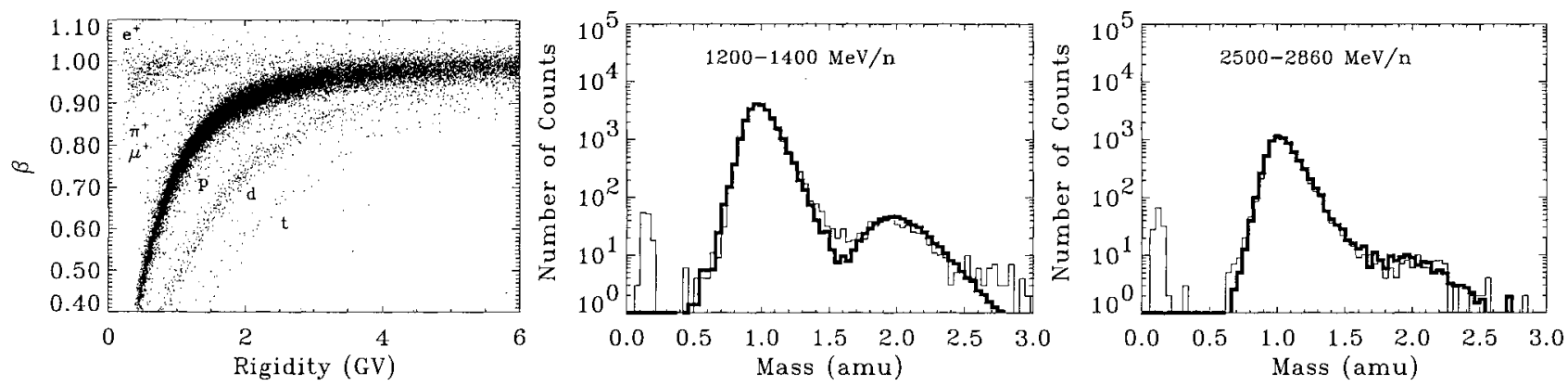

FIGURE 1. IMAX mass separation for $Z=1, \beta=1$ particles along with the corresponding mass histograms in two separate energy intervals. The mass is determined between $2.5-2.860 \mathrm{GeV} /$ nucleon using the velocity obtained from the Cherenkov counters. The thick solid lines represent an instrument simulation, while the thin solid lines refer to measured distributions.

IMAX flew in July, 1992 from Lynn Lake, Manitoba, Canada. The flight lasted 16 hours at float including a long ascent of $\sim 7$ hours. IMAX reached a maximum float altitude of $36 \mathrm{~km}\left(5 \mathrm{~g} / \mathrm{cm}^{2}\right.$ of residual atmosphere). The geomagnetic cutoff varied between $0.35 \mathrm{GV}$ at Lynn Lake and $0.63 \mathrm{GV}$ at Peace River, Alberta.

\section{DATA ANALYSIS}

IMAX events are accepted based on a four-fold coincidence between the photomultiplier signals from the opposite sides of the top and bottom TOF scintillators. The selection criteria employed to obtain a clean sample of charge one particles are discussed in (1).

Figure 1 shows the isotopic separation for charge one particles using the $\beta$-rigidity technique. The corresponding mass histograms in two representative energy intervals are shown in Figure 1, where the velocity in the higher energy interval is obtained from the aerogel Cherenkov counter. Due to the non-gaussian behavior of the distributions, a simulation was developed to accurately model the instrument response (6),(7). The simulation takes into account, on an event by event basis, the actual spectral shape of the incoming particles, the TOF timing resolution, the photoelectron statistical fluctuations and $\delta$-ray contributions to the Cherenkov light yield. It also takes into account the spatial resolution and rigidity resolution of the tracking system, and the effects of multiple coulomb scattering. The simulation results are shown in Figure 1 as the thick solid lines.;

\section{RESULTS}

In order to determine the flux of deuterium at the top of the atmosphere, it is necessary to account for nuclear interaction losses within in the instrument and atmosphere as well as for the secondary population of ${ }^{2} \mathrm{H}$ produced from the interaction of protons and helium in the $5 \mathrm{~g} / \mathrm{cm}^{2}$ residual atmosphere above the instrument. The attenuation of ${ }^{2} \mathrm{H}$ within the instrument and atmosphere is determined using a universal parametrization for the total reaction cross section given by Tripathi et al. (10). This model is in good agreement with current measurements for the inelastic cross sections of ${ }^{2} \mathrm{H}+\mathrm{p}$ and ${ }^{3} \mathrm{He}+\mathrm{p}$ reactions (10), (11). IMAX has a mean grammage in the instrument of $13.8 \mathrm{~g} / \mathrm{cm}^{2}$. The different materials encountered during the particle's traversal through the IMAX instrument are accounted for in this calculation.
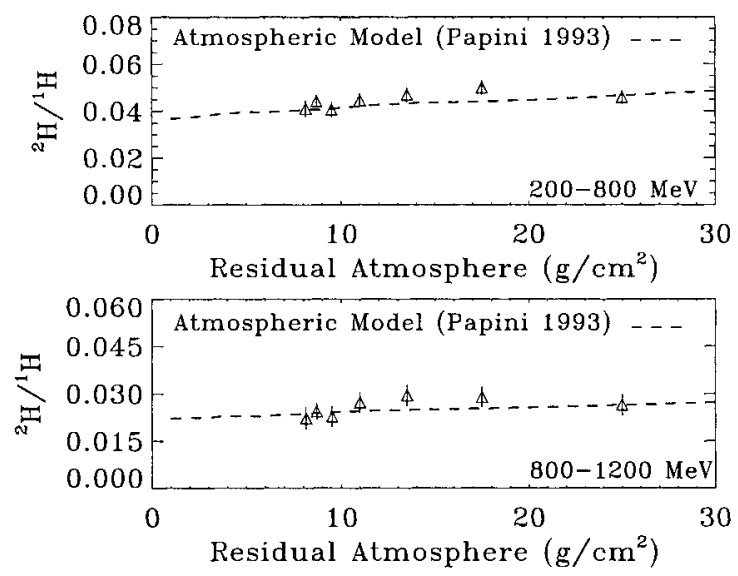

FIGURE 2. IMAX ${ }^{2} \mathrm{H} /{ }^{1} \mathrm{H}$ as a function of atmospheric depth in two separate energy intervals. The model of Papini is normalized to the IMAX data as discussed in the text.

The contribution from atmospheric secondary ${ }^{2} \mathrm{H}$ is determined from calculations by Papini et al. (4),(5). Several other calculations have been performed including those of Webber (12) and Lijowski et al. (3). Current calculations differ by as much as a factor of 2-3 where the differences are most likely due to the assumed pri- 
mary proton and helium spectra and the assumed interaction cross sections. In order to better constrain our estimate of the contribution of secondary ${ }^{2} \mathrm{H}$, we obtain the ${ }^{2} \mathrm{H} /{ }^{1} \mathrm{H}$ ratio as a function of depth in the atmosphere during IMAX's long $\sim 7$ hour ascent to float altitudes. Figure 2 shows the ${ }^{2} \mathrm{H} /{ }^{1} \mathrm{H}$ ratio at seven separate depths in the atmosphere. The dashed curve is the calculation by Papini (4) for the production of secondary deuterium in $5 \mathrm{~g} / \mathrm{cm}^{2}$ residual atmosphere during solar minimum conditions. The Papini calculations are fit to the IMAX ${ }^{2} \mathrm{H} /{ }^{1} \mathrm{H}$ ratio as a function of depth resulting in a top of the atmosphere ratio of $0.036 \pm .004$ at $600 \mathrm{MeV} /$ nucleon and $0.022 \pm .003$ at $1 \mathrm{GeV} /$ nucleon, as shown in Figure 2. The contribution of secondary deuterium predicted by Papini's model is consistent with the rate of growth of ${ }^{2} \mathrm{H} /{ }^{1} \mathrm{H}$ as a function of depth in the atmosphere measured by IMAX. Secondary deuterium produced within the instrument is vetoed by the instrument trigger and event selection criteria.

\section{DISCUSSION AND CONCLUSION}

Figure 3 shows the ${ }^{2} \mathrm{H}$ spectrum at the top of the atmosphere along with recent measurements from the BESS experiment during three separate flights from 1993 to 1995 (11). The solar modulation during the IMAX 1992 flight is consistent with a modulation parameter in the spherically symmetric force-field model of $\phi=750 \mathrm{MV}$ (1). At low energies, where the effects of atmospheric

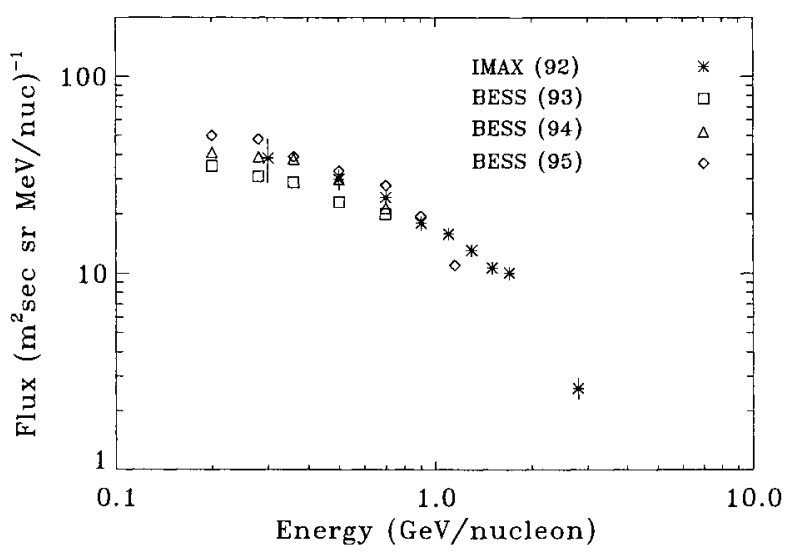

FIGURE 3. IMAX top of the atmosphere ${ }^{2} \mathrm{H}$ flux compared with measurements from the BESS experiment (11).

secondaries are significant, IMAX observes a higher deuterium flux than BESS observations during 1993, contrary to what one might expect from solar modulation effects alone. The discrepancy appears to be in the choice of the absolute value for the calculated contribution of atmospheric secondary deuterium. We find the IMAX growth curves are consistent with Papini's atmospheric secondary calculation at solar minimum conditions. On the other hand, the BESS secondary calculations result in fluxes a factor of $\sim 2$ lower, closer to Papini's solar maximum calculation (11). Table 1 lists the IMAX measurements for the ${ }^{2} \mathrm{H}$ flux at the top of the instrument and atmosphere.

Table $1 .{ }^{2} \mathrm{H}$ Flux at the top of the instrument (TOI) and atmosphere(TOA).

\begin{tabular}{cccc}
\hline $\begin{array}{c}\text { Energy } \\
(\mathrm{GeV} / \mathrm{n})\end{array}$ & $\begin{array}{c}\text { Mean Energy } \\
(\mathrm{GeV} / \mathrm{n})\end{array}$ & ${ }^{2} \mathrm{H}_{T O I}$ & ${ }^{2} \mathrm{H}_{T O A}$ \\
$0.4-0.6$ & 0.5 & $35.8 \pm 2.2$ & $30.0 \pm 3.5$ \\
$0.6-0.8$ & 0.7 & $25.5 \pm 1.5$ & $24.3 \pm 1.9$ \\
$0.8-1.0$ & 0.9 & $18.3 \pm 1.2$ & $18.0 \pm 1.5$ \\
$1.0-1.2$ & 1.0 & $15.5 \pm 1.0$ & $15.8 \pm 1.1$ \\
$1.2-1.4$ & 1.2 & $12.8 \pm 0.8$ & $13.1 \pm 0.9$ \\
$1.4-1.6$ & 1.5 & $10.4 \pm 0.7$ & $10.7 \pm 0.7$ \\
$1.6-1.8$ & 1.7 & $9.7 \pm 0.7$ & $10.1 \pm 0.7$ \\
$2.5-2.9$ & 2.7 & $2.6 \pm 0.3$ & $2.6 \pm 0.3$ \\
\hline
\end{tabular}

The ratios of ${ }^{2} \mathrm{H} /{ }^{1} \mathrm{H}$ and ${ }^{2} \mathrm{H} /{ }^{3} \mathrm{He}$ are shown in Figures $4[\mathrm{a}]$ and $4[\mathrm{~b}]$ along with the predictions of propagation models based on a standard Leaky Box calculation by Seo et al. (9) and a reacceleration model by Seo \& Ptuskin (8). The ${ }^{2} \mathrm{H} /{ }^{1} \mathrm{H}$ ratio is in excess of the model predictions at low energies where the atmospheric secondary contribution is largest. The IMAX results for the ${ }^{2} \mathrm{H} /{ }^{3} \mathrm{He}$ ratio are in better agreement at higher energies, not unexpectedly, since the ${ }^{2} \mathrm{H} /{ }^{3} \mathrm{He}$ ratio is essentially independent of pathlength in the interstellar medium.

A measure of the ${ }^{2} \mathrm{H} /{ }^{4} \mathrm{He}$ ratio over a wide energy range may help to distinguish between existing propagation models, especially as we expect the ${ }^{2} \mathrm{H} /{ }^{4} \mathrm{He}$ ratio to exhibit a strong energy dependence resulting from the ${ }^{2} \mathrm{H}$ production cross sections. Reacceleration, on the other hand, would smear out this energy dependence. The ${ }^{2} \mathrm{H} /{ }^{4} \mathrm{He}$ and ${ }^{3} \mathrm{He} /{ }^{4} \mathrm{He}$ ratios are shown in Figures $4[\mathrm{c}]$ and $4[\mathrm{~d}]$ and are compared with predictions from Seo \& Ptuskin (8), Webber (14) and Reimer et al. (6). Webber's calculation is a standard Leaky Box Model with a pathlength $\lambda=31.6 \beta \mathrm{R}^{-0.6}$ for $\mathrm{R}>4.7 \mathrm{GV}$ and $\lambda=1.2 .5 \beta$ below $4.7 \mathrm{GV}$ that is based on $\mathrm{B} / \mathrm{C}$ measurements (15). The solid curve in Figure 4[c] is a calculation by Seo \& Ptuskin that includes the affects of reacceleration (8). Finally, the solid curve in Figure 4[d] is a standard Leaky Box calculation by Reimer et al. (6) that assumes similar input parameters to the Webber calculations (14), though with slightly different cross sections.

The IMAX observations of the ${ }^{2} \mathrm{H}$ are generally consistent with predictions of the standard Leaky Box Model in which protons and helium have the same propagation history as the heavier component of cosmic rays. How- 

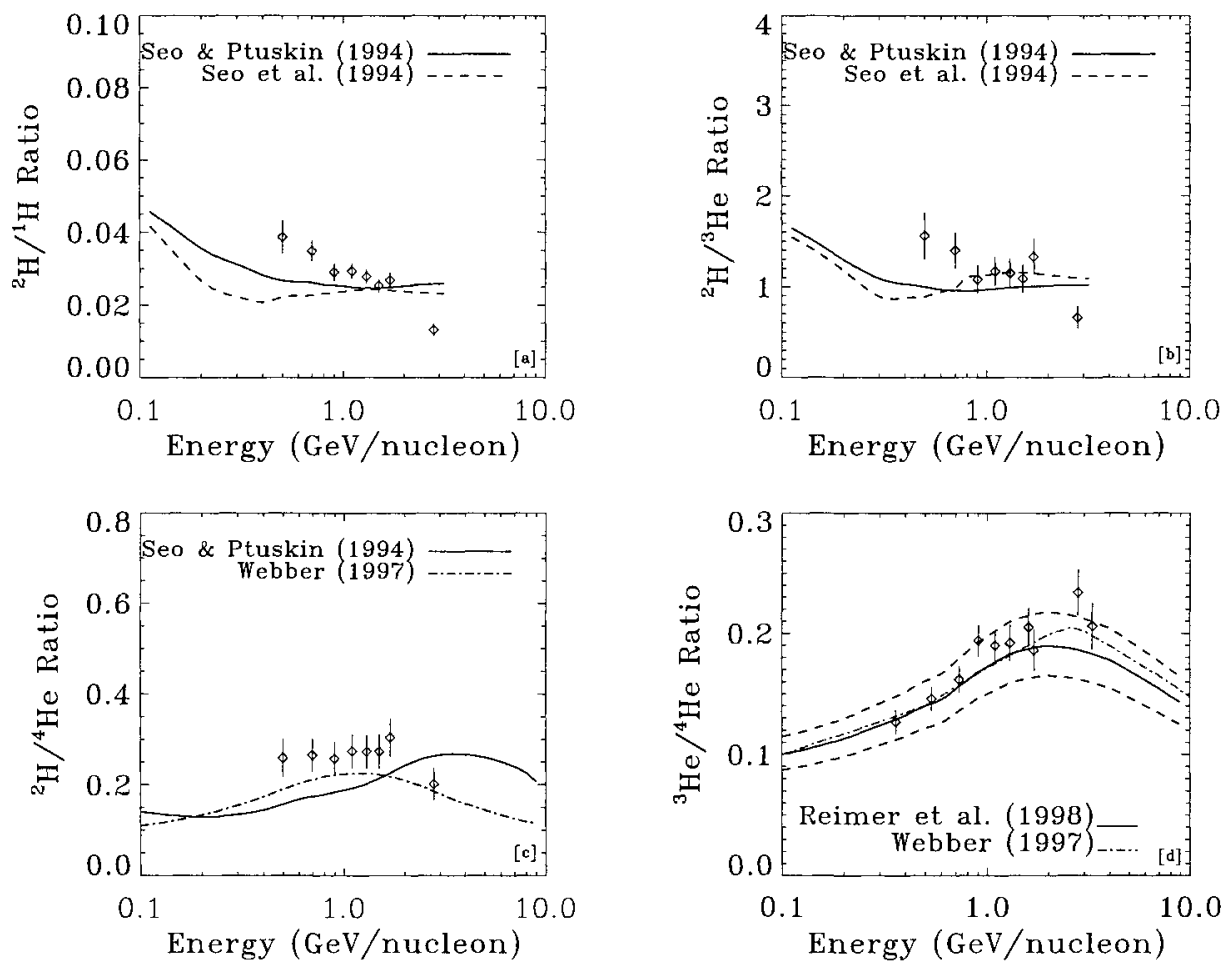

FIGURE 4. [a]-[b]: IMAX ${ }^{2} \mathrm{H} /{ }^{1} \mathrm{H}$ and ${ }^{2} \mathrm{H} /{ }^{3} \mathrm{He}$ ratios compared with the propagation model with reacceleration of Seo \& Ptuskin (8) (solid curve) and the standard Leaky Box calculation of Seo et al. (9) (dashed curve), [c]-[d]: ${ }^{2} \mathrm{H} /{ }^{4} \mathrm{He}$, and ${ }^{3} \mathrm{He} /{ }^{4} \mathrm{He}$ ratios compared with propagation models of Webber (14) (dot-dashed curve), and Reimer et al. (6) (solid curve). The dashed curves in [d] represent the uncertainties in the Reimer et al. calculation.

ever, there is a tendency for the ${ }^{2} \mathrm{H} /{ }^{4} \mathrm{He}$ and ${ }^{2} \mathrm{H} /{ }^{1} \mathrm{H}$ observations to lie somewhat above the model predictions. Understanding this possible excess will require a thorough investigation of the dependence of these ratios on the relevant production cross sections for ${ }^{2} \mathrm{H}$ and on the assumed pathlength distribution.

\section{ACKNOWLEDGMENTS}

The IMAX project was supported in the United States by NASA under NAG5-5227 (Caltech) and under RTOP 353-87-02 (GSFC) and grants NAGW-1418 (NMSU/BBMF) and in Germany by the Deutsche Forschungsgemeinschaft (DFG) and the Bundesministerium für Bildung, Wissenschaft, Forschung und Techologie (BMBF).

\section{REFERENCES}

1. Menn, W., et al., accepted for publication in $A p J, \mathbf{5 3 3}$, No. $1,(2000)$.

2. Hof, M., et al., Nucl. Inst. \& Meth., A345, (1994), 561.
3. Lijowski, M., (1994), Ph.D. Dissertation, Louisiana State University.

4. Papini, P., et al., Proc. 23rd Int. Cosmic Ray Conf. (Calgary), (1993), 503.

5. Papini, P., et al., Il Nuovo Cimento, 19, (1996), 367.

6. Reimer, O., et al., ApJ, 496, (1998) 490.

7. Reimer, O., et al., Proc. 24th Int. Cosmic Ray Conf. (Rome), 2, (1995), 614.

8. Seo, E.S., and Ptuskin, V.S., ApJ, 431, (1994), 705.

9. Seo, E.S., et al., ApJ, 432, (1994), 656.

10. Tripathi, R.K., et al., NASA/TP-1999-209726, NASA Langley Research Center, (1999).

11. Wang, J.Z., et al., Proc. 26th Int. Cosmic Ray Conf. (Salt Lake City) 3, (1999), 37.

12. Webber, W.R., et al., ApJ, 380, (1991), 230.

13. Webber, W.R., et al., ApJ, 392, (1992), L91.

14. Webber, W.R., Adv. Space Res., 19, No. 5, (1997), 755.

15. Webber, W.R., et al., ApJ, 457, (1996), 435. 